d'œufs et de laitance, soit dans des incubateurs ordinaires, soit, de préférence, dans des carafes de Zug contenant de l'eau. Après avoir remué les œufs pendant quelques secondes avec une plume, on met en marche les appareils et on règle le débit de l'eau.

L'incubation doit être faite de préférence avec une eau dont la température est comprise entre Io et 15 degrés centigrades.

L'incubation dans les carafes de Zug présente le grand avantage d'empécher les œufs sains d'entrer en contact avec les oufs qui, ayant échappé à la fécondation, s'altèrent rapidement. Toute contamination des ceufs sains est ainsi évitée. Toutefois il est bon, pour éviter un certain déchet au moment de l'éclosion, d'interrompre l'incubation dans les carafes de Zug à l'approche de l'éclosion ou, au plus tard, au début de celle-ci.

Les œufs, sont alors disposés, soit directement sur le fond des bacs d'alevinage, soit, de préférence, sur des tamis à mailles très fines (Imm. au maximum) suspendus dans les bacs à une faible distance du niveau de l'eau.

Lorsque l'éclosion est terminée, on procède à un bon nettoyage pour enlever les œufs morts et les débris de coque des cufs. Il est utile de placer alors, dans les bacs d'alevinage, des végétaux aquatiques (Fontinalis Myriophyllum, Renoncules aquatiques, etc...) qui offrent aux alevins d'excellents points d'appui pendant leur période de vie fixée.

Lorsqu'au bout d'une dizaine de jours les alevins se détachent, le moment est venu, en s'entourant des précautions nécessaires pour ne pas blesser ces êtres fragiles, de procéder au lancement. Toutefois, si l'on dispose d'une nourriture naturelle appropriée, en particulier d'un élevage de Daphnies, on peut essayer de poursuivre plus avant l'alevinage en faisant 3 ou 4 distributions de nourriture par jour. Mais il sera prudent de sélectionner les sujets dès que des inégalités de taille commenceront à s'accuser, afin d'éviter les pertes par cannibalisme qui ne manqueraient pas de se produire.

\title{
DE QUELQUES ESSAIS DE SAUMON DE FONTAINE DANS LES PYRÉNÉES
}

\author{
(Salvelinus fontinalis Mitchill)
}

\author{
Par W. J. LARRIEU
}

Inspecteur principal des Enux et Forêts, Oloron.

On est frappé, en prospectant les eaux pyrénéennes, de constater qu'il existe, dans les têtes de bassin de l'Adour et des Gaves, de très nombreux tronçons de rivières, dont la longueur atteint parfois 6 kilomètres, absolument dépourvus de poissons, alors qu'à première vue riẹn ne semble s'oppo- 


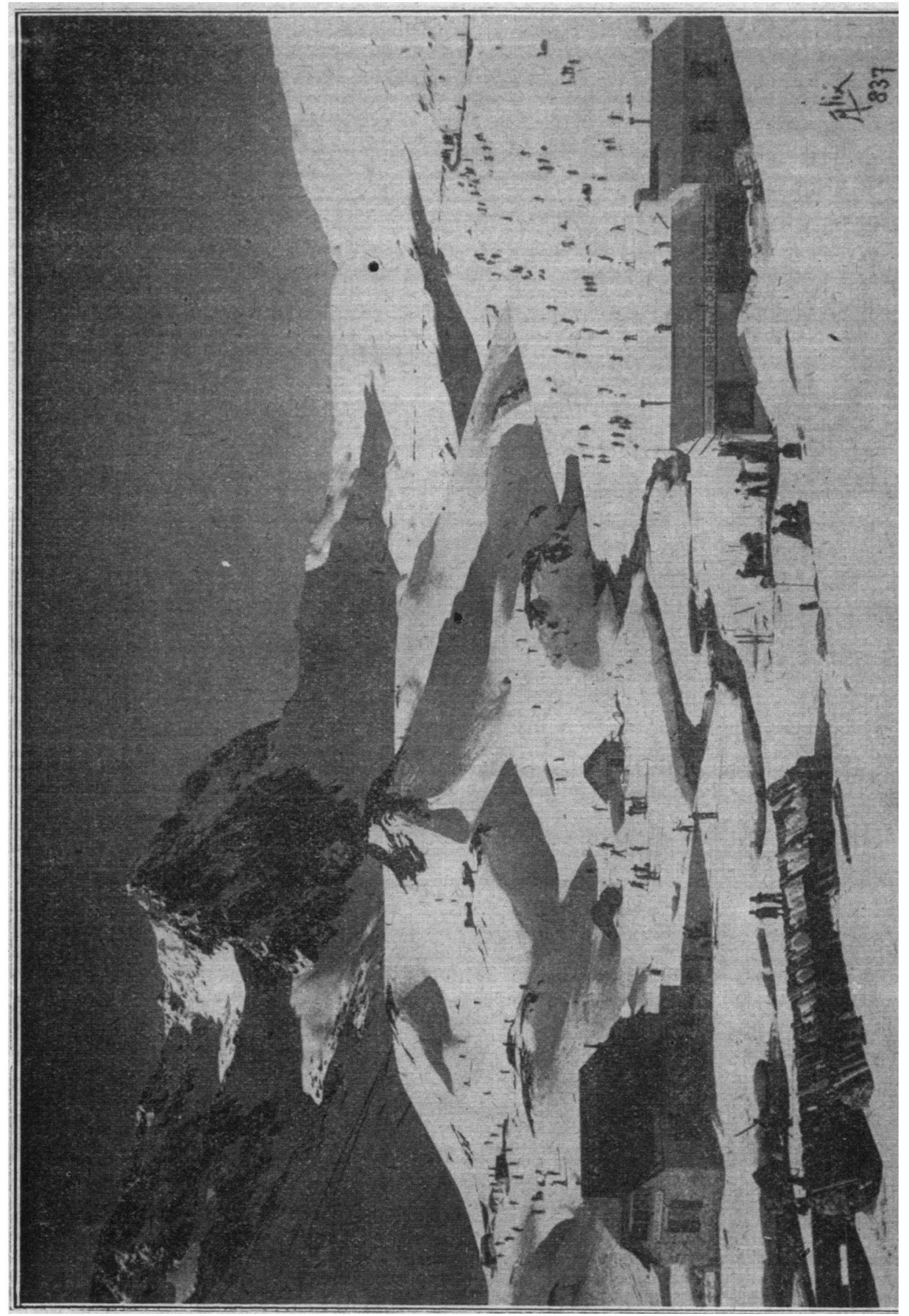

हัّ 
ser à la vie piscicole. On trouve, en effet, en grand nombre, même aux hautes altitudes voisines de 2.000 mètres, des larves de Phrygane, Ecdyures, et autres espèces rhéophiles, même des têtards de Grenouille qui pourraient fournir une nourriture abondante aux poissons carnivores. Par ailleurs, le $\mathrm{pH}$. est favorable et la quantité d'oxygène dissous satisfaisante (r).

En général, ces têtes de bassin se présentent sous forme de rivières à pente moyenne coulant sur des platcaux séparés des basses vallées par des cascades plus ou moins hautes.

Nos observations ont porté sur les rivières suivantes :

Haut Gave de Pau ;

Adour du Tourmalet ;

Adour de Tramezaygues ;

Gave de Lescun (affluent rive gauche du Gave d'Aspe) ;

Ruisseau la Brennère (affluent du Gave de Lescun) ;

Haut Gave d'Aspe ;

Gave de Grourette (affluent du Gave d'Ossau) ;

Vert d'A rette (affluent du Gave d'Oloron);

Haut Gave d'Ossau.

Dans tous ces cours d'eau, à partir d'un certain point, marqué en général par des cascades plus ou moins élevées, et à altitude variant de i 5 à r.80o mètres, la Truite n'existe plus.

Le cas le plus caractéristique est celui de l'Adour.

Ce cours d'eau, qui prend sa source dans la haute Vallée de Campan, est formé de trois branches qui se réunissent sur le plateau d'Artigues : l'Adour du Tourmalet, - l'Adour de Caderolles — et l'Adour de Tramezaygues.

Avant d'atteindre le plateau d'Artigues, ces trois rivières franchissent des rapides qui atteignent une vingtaine de mètres. Mais, tandis que l'Adour de Caderolles présente les cascades les plus élevées, il est le seul à avoir de la Truite, ef celle-ci y est abondante. Les deux autres en sont dépourvus.

Ceci tient-il à la nature des terrains traversés ?

L'Adour de Caderolles prend sa source dans la chaîne centrale, en terrain granitique, tandis que les deux autres naissent dans les schistes et micaschistes du Tourmalet et du Pic dı Midi de Bigorre. A première vue, ceci ne paraît pas avoir d'importance.

Au dire des bergers qui fréquentent les pâturages du Tourmalet, des pêcheurs auraient essayé, à plusieurs reprises, d'introduire la Truite dans l'Adour en y apportant des adultes. Le résultat avait été négatif et l'on estimait dans le pays que la Truité ne pouvait vivre à cette altitude.

(r) Analyse de l'eau de l'Adour du Tourmalet, le 28 Juillet 1936 : température : $6^{\circ}$ centigrades a 5 heures par temps brumeux, pH. 7,3 - oxygène dissous : 6 mc, 700 ). 
Après avoir pris. l'avis de $M$. le Professeur Lécer nous avons essayé, au cours de l'été rg34, de faire quelques déversements de Saumon de fontaine dans les cinq rivières suivantes :

Adour du Tourmalet ;

Gave de Lescun ;

Haut Gave d'Aspe ;

Gave de Gourette ;

Vert d'A rette.

Les alevins introduits provenant de l'Etablissement Domanial d'Oloron, étaient âgés de 5 à 6 mois et de belle taille. L'immersion a été faite très lentement de manière à amener les poissons à passer sans brusque transition d'une eáu de 12 à $13^{\circ}$ - celle des bidons de transport - à l'eau de la rívière à température très basse.

Dès l'été rg35, il apparaissait que ces ałevins avaient parfaitement vécu et prospéré ; - ils atteignaient de 12 à $\mathrm{I} / 4$ centimètres.

Un an après, dès l'ouverture de la pêche en ig36, il était annoncé qu'on prenait des sujets nombreux, de belle taille et se défendant bien ; - chair rose et savoureuse - mais de conservation difficile. Nous avons pu vérifier personnellement les captures suivantes :

$\mathrm{I}^{\circ}$ Le $\mathrm{r} 6$ Juin 1936 , dans le Vert d'Arette : 4 sujets, âgés de 2 ans $1 / 2$, 2 mâles et 2 femelles, pesant au total 850 grammes. Le plus gros, un mâle, pesait 240 grammes et mesurait 28 centimètres de longueur totale. Les testicules étaient parfaitement développés. Chez les deux femelles, les ovaires étaient également bien formés et présentaient des œufs de I $\mathrm{mm}$. de diamètre environ.

$2^{\circ}$ Le 20 Juin 1936 , dans le Gave de Lescun, 4 sujets d'environ r5o grammes chacun, sexe inconfru, les poissons n'ayant pas été ouverts devant nous.

$3^{\circ}$ Le 28 Juillet 1936 , dans l'Adour du Tourmalet, 3 femelles pesant ensemble 350 grammes dont l'une 160 grammes - ovaires développés.

Tous ces sujets avaient été immergés au cours de l'été 1934.

$4^{\circ}$ Le 6 Juin 1937 , dans le Gave d'Ossau, 4 sujets dont un male pesant 320 grammes - les 3 autres compris entre 2 io et 240 grammes. La première immersion datait de l'étế 1935 - ils n'étaient donc agés que de 2 ans $1 / 2$.

Enfin, il nous a été signalé qu'un sujet pris en Aout rg37 dans l'Adour du Tourmalet par le Maréchal des Logis de Gendarmerie de Campan pesait 430 grammes - il était au plus agé de 3 ans $1 / 2$ (immersion de r934).

Devant ces résultats encourageants, nous avons continué nos immersions en $1935-1936$ et $1_{93}$ et les avons étendues peu à peu dans ces eaux de haute altitude.

Une question pourtant se posait. 
Le Saumon de fontaine avait prospéré dans ces eaux froides d'une façon presque surprenante - mais allait-il se reproduire ?

Les glandes génitales sur les sujets disséqués nous avaient paru normalement développées - mais aucune ponte n avait été observée.

C'est seulement au cours d'Octobre ${ }^{1} 9^{3} 7$ que la fraie fut signalée comme commencée dans l'Adour du Tourmalet. Nous y étant rendu dans l'aprèsmidi du I $^{\text {er }}$ Novembre, nous avons pu constater la ponte d'une femelle fécondée par deux mâles, nơn dans le lit de la rivière, mais sur un petit ruisseau qui s'y déverse. Ces 3 reproducteurs étaient encore petits et ne devaient être âgés que de 2 ans.

Avant la crue du 28 Octobre, on voyait, m'a-t-on affirmé, le poisson frayer dans le lit même du cours d'eau.

Notre observation n'a pu porter que sur un nombre restreint de sujets, mais elle est à retenir.

Un autre indice paraît prouver que la fraie a eu lieu dans le Gave d'Ossau au cours de l'hiver $1936-19^{3} 7$ après 2 ans d'immersion. Deux des sujèts capturés le 6 Juin 1937 avaient, en effet, dans la bouche plusieurs petits alevins - mais déjà trop mal en point pour pouvoir affirmer que c'étaient des alevins de Salvelinus. Il ne semble pas, toutefois, qu'il puisse y avoir de doute, étant donné que la Truite n'avait pas été signalée dans ces paraegs.

De ces premières constatations, il semble bien résulter que le saumon de fontaine s'adapte entièrement et très facilement à des eaux très froides, où la Truite paraît ne pas vivre ou du moins ne pas se reproduire.

Les sujets provenant des premières immersions ont grossi d'une façon que nous pensons exceptionnelle. Arrivés dans des eaux non peuplées, ils y ont trouvé une nourriture abondante qu'ils ont absorbée avec voracité, Mais cette voracité, déjà signalée par les pisciculteurs, n'est pas exempte de cannibalisme.

Outre les alevins trouvés dans la bouche des sujets pris dans le Gave d'Ossau en 1937 , nous avons pu constater que les Saumons de fontaine, capturés dans le Vert d'A rette en 1936 , avaient ingurgité, chacun, 5 à 6 alevins de la même espèce provenant d'un déversement fait quelques heures auparavant dans les mêmes parages (circonstance à retenir au moment des immersions).

Les essais que nous avons tentés sont encore trop récents pour qu'on puisse en tirer une conclusion définitive. Il semble bien cependant, après 3. années d'expérience, qu'on peut espérer au moyen du Saumon de fontaine peupler et rendre productifs les cours d'eau pyrénéens de haute altitude jusqu'ici considérés comme impropres à la vie du poisson. 